Article

\title{
Investigation on the Flow in a Rotor-Stator Cavity with Centripetal Through-Flow ${ }^{\dagger}$
}

\author{
Bo $\mathrm{Hu}^{*}$, Dieter Brillert, Hans Josef Dohmen and Friedrich-Karl Benra \\ Department of Mechanical Engineering, University of Duisburg-Essen, 47057 Duisburg, Germany; \\ dieter.brillert@uni-due.de (D.B.); hans-josef.dohmen@uni-due.de (H.J.D.); friedrich.benra@uni-due.de (F.-K.B.) \\ * Correspondence: bo.hu.1987@stud.uni-due.de; Tel.: +49-203-379-1703 \\ + This paper is an extended version of our paper in Proceedings of the European Turbomachinery Conference \\ ETC12, 2017, Paper No. 97.
}

Academic Editor: Claus Sieverding

Received: 31 July 2017; Accepted: 6 October 2017; Published: 19 October 2017

\begin{abstract}
Daily and Nece distinguished four flow regimes in an enclosed rotor-stator cavity, which are dependent on the circumferential Reynolds number and dimensionless axial gap width. A diagram of the different flow regimes including the respective mean profiles for both tangential and radial velocity was developed. The coefficients for the different flow regimes have also been correlated. In centrifugal pumps and turbines, the centripetal through-flow is quite common from the outer radius of the impeller to the impeller eye, which has a strong influence on the radial pressure distribution, axial thrust and frictional torque. The influence of the centripetal through-flow on the cavity flow with different circumferential Reynolds numbers and dimensionless axial gap width is not sufficiently investigated. It is also quite important to convert the 2D Daily and Nece diagram into $3 \mathrm{D}$ by introducing the through-flow coefficient. In order to investigate the impact of the centripetal through-flow, a test rig is designed and built up at the University of Duisburg-Essen. The design of the test rig is described. The impact of the above mentioned parameters on the velocity profile, pressure distribution, axial thrust and frictional torque are presented and analyzed in this paper. The 3D Daily and Nece diagram introducing the through-flow coefficient is also organized in this paper.
\end{abstract}

Keywords: rotor-stator cavity; centripetal through-flow; axial thrust; frictional torque

\section{Introduction}

In radial pumps and turbines, the leakage flow (centripetal through-flow) is quite common from the outer radius of the impeller to the impeller eye, which has a major impact on the pressure distribution, axial thrust $\left(F_{a}\right)$ and frictional torque. Von Kármán [1] and Cochran [2] gave a solution of the ordinary differential equation for the steady, axisymmetric, incompressible flow. Daily and Nece [3] examined the flow of an enclosed rotating disk both analytically and experimentally. They distinguished the four flow regimes, shown in Figure 1, by correlating different empirical equations of the moment coefficients. Kurokawa et al. [4-6] studied the cavity flow with both centrifugal and centripetal through-flow. Schlichting and Gersten [7] organized an implicit relation based on the results of Goldstein [8] for the moment coefficient under turbulent flow conditions. Poncet et al. [9] studied the centripetal through-flow in a rotor-stator cavity and obtained an equation of the core swirl ratio $K$ based on the local flow rate coefficient $\left(C_{q r}\right)$ for Batchelor type flow [10]. For Batchelor type flow, the centrifugal disk boundary layer and the centripetal wall boundary layer are separated by a central core. Debuchy et al. [11] derived an explicit equation for $K$ which is valid over a wide range of $C_{q r}$. Launder et al. [12] provided a review of the current understanding of instability pattern that are created in rotor-stator cavities leading to transition and eventually turbulence. Recent experimental 
investigations up to circumferential Reynolds numbers $R e=5 \times 10^{8}$ with and without through-flow have been conducted by Coren et al. [13], Long et al. [14] and Barabas et al. [15]. The scope of the present study is shown in Figure 1.

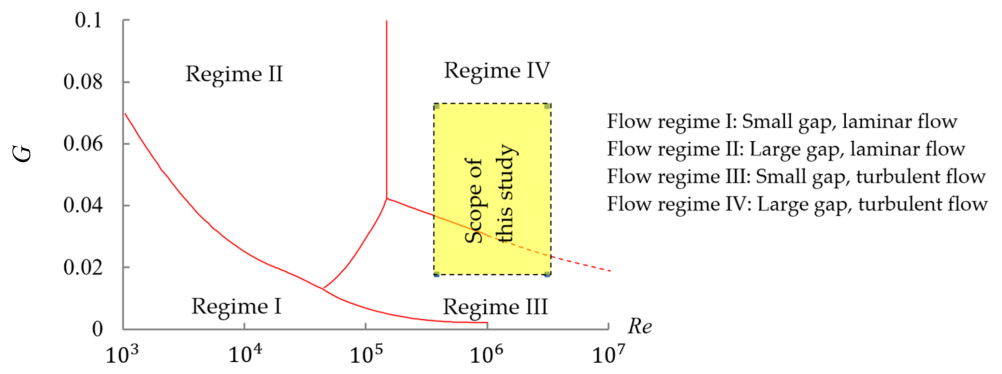

Figure 1. Flow regimes according to Daily and Nece [3].

The main dimensions of the rotor-stator cavity are illustrated in Figure 2. This study focuses on the influence of non-pre-swirl centripetal through-flow on the cavity flow. Based on the results from previous studies, $C_{D}{ }^{\prime}$ (through-flow coefficient) may have a large influence on the moment coefficient, noted as $C_{M}$. Uncertainties still exist in the effect of $C_{D}{ }^{\prime}$ on $C_{M}$ with different values of $\operatorname{Re}$ and $G$ (dimensionless axial gap). This study is aimed to provide more results to increase the dataset and to better understand the influence of above parameters on $C_{p}$ (pressure coefficient), $C_{F}$ (axial thrust coefficient) and $C_{M}$. The definitions of the significant dimensionless parameters in this study are given in Equations (1a)-(1k).

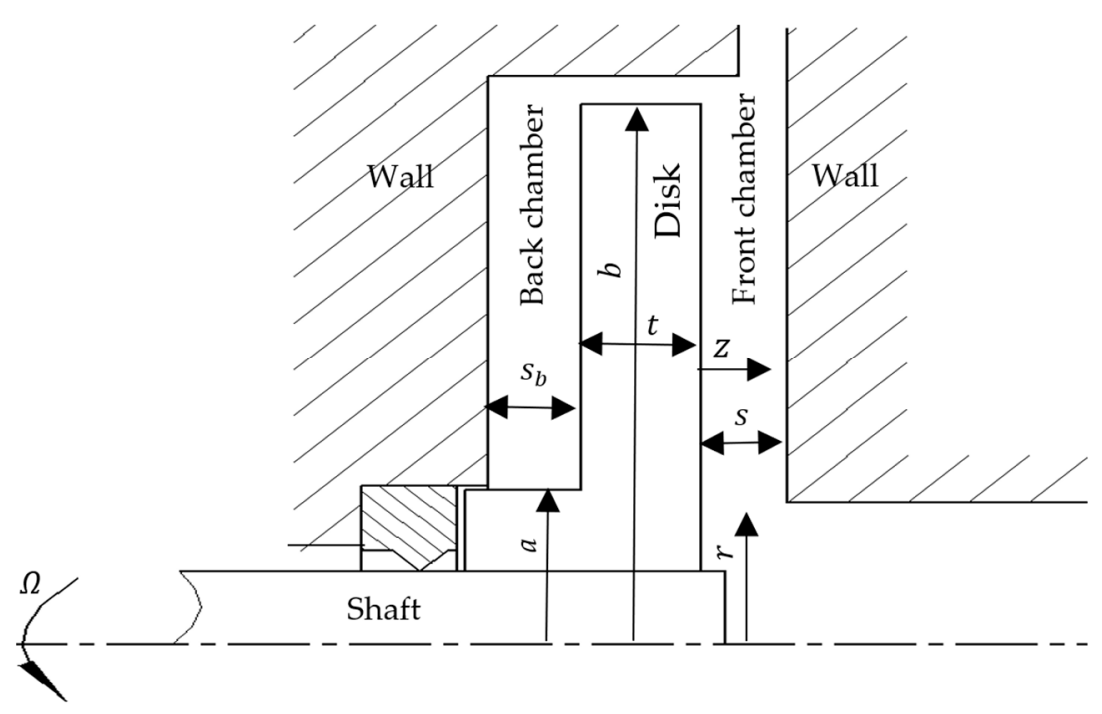

Figure 2. Main dimensions of the test rig. $a$ : Hub radius; $b$ : outer radius of the disk; $r$ : radial coordinate; $s$ : axial gap of the front chamber; $s_{b}$ : axial gap of the back chamber; $t$ : thickness of the disk; $z$ : axial coordinate.

$$
\begin{aligned}
R e & =\frac{\Omega \cdot b^{2}}{v} \\
R e_{\varphi} & =\frac{\Omega \cdot r^{2}}{v} \\
G & =\frac{s}{b}
\end{aligned}
$$




$$
\begin{gathered}
C_{D}{ }^{\prime}=\frac{\dot{m}}{\mu \cdot b} \\
C_{F}=\int_{a}^{b} \frac{2 \cdot \pi \cdot\left(p_{b}-p\right) \cdot r \mathrm{~d} r}{\rho \cdot \omega^{2} \cdot b^{4}} \\
C_{q r}=\frac{Q \cdot R e_{\varphi}^{0.2}}{2 \cdot \pi \cdot \Omega \cdot r^{3}} \\
\zeta=\frac{z}{s} \\
x=\frac{r}{b} \\
C_{M}=\frac{2 \cdot|M|}{\rho \cdot \Omega^{2} \cdot b^{5}} \\
p^{*}=\frac{p}{\rho \cdot \Omega^{2} \cdot b^{2}} \\
C_{p}=p^{*}(x=1)-p^{*}(x)
\end{gathered}
$$

\section{Theoretical Analysis}

In this study, the values of $Q$ (volumetric through-flow rate), $C_{D}{ }^{\prime}$ and $C_{q r}$ are negative for centripetal through-flow. Using a two-component Laser Doppler Anemometer (LDA) system, Poncet et al. [9] correlated Equation (2a) to evaluate the core swirl ratio $K$ (the ratio of the angular velocity of the fluid to that of the disk at $\zeta=0.5$ ) with centripetal through-flow when $C_{q r} \geq-0.2$. Debuchy et al. [11] determined Equation (2b) to calculate the values of $K$ for a wider range $C_{q r} \geq-0.5$ with a two-component LDA system. The results from Equation (2b) are smaller than those from Equation (2a) at large values of $\left|C_{q r}\right|$, compared in Figure 3.

$$
\begin{gathered}
K=2 \cdot\left(-5.9 \cdot C_{q r}+0.63\right)^{\frac{5}{7}}-1 \\
K=\left[\frac{-8.85 \cdot C_{q r}+0.5}{e^{\left(-1.45 C_{q r}\right)}}\right]^{\frac{5}{4}}
\end{gathered}
$$

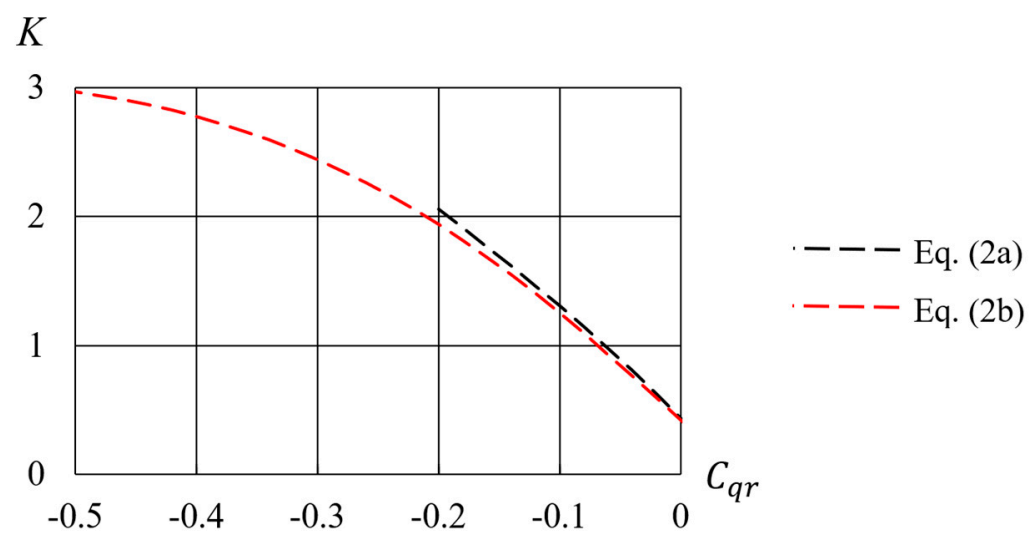

Figure 3. Comparison of results from Equations (2a) and (2b). K: core swirl ratio at $\zeta=0.5 ; C_{q r}$ : local flow rate coefficient; Eq: equation.

A number of studies, such as those by Kurokawa et al. [6], Poncet et al. [9], Coren et al. [13], and Barabas et al. [15], show that the pressure distribution along the radius of the disk can be 
estimated with the core swirl ratio $K$ both with and without through-flow. Will et al. [16-18] determined Equation (3) to evaluate the pressure distribution along the radius of the disk for the incompressible, steady flow. It is obtained directly from the radial momentum equation when the turbulent shear stress is neglected. In a rotor-stator cavity, the cross sectional area changes in radial direction. Consequently, the pressure must also change since the mean velocity changes in radial direction according to the continuity equation.

$$
\frac{\partial p}{\partial r}=\rho\left(\frac{\mathrm{v}_{\varphi}{ }^{2}}{r}-\mathrm{v}_{r} \frac{\partial \mathrm{v}_{r}}{\partial r}\right)=\rho \cdot K^{2} \cdot \Omega^{2} \cdot r+\frac{\rho \cdot Q^{2}}{4 \cdot \pi^{2} \cdot s^{2} \cdot r^{3}}
$$

The difference of the force on both sides of the disk is the main source for axial thrust, noted as $F_{a}$, calculated with Equation (4a). $F_{a f}$ (force on the front surface of the disk; calculated with Equation (4b)) and $C_{F f}\left(C_{F}\right.$ on the front surface) respectively represent the force and the thrust coefficient on the front surface of the disk (in the front chamber, shown in Figure 2), while $F_{a b}$ (force on the back surface of the disk; calculated with Equation (4c)) and $C_{F b}$ are those on the back surface of the disk (in the back chamber). $a$ and $p_{b}$ represent the radius of the hub (see Figure 2) and the pressure at $x=1$, respectively. The back chamber $(G=0.072)$, shown in Figure 2, is supposed to be an enclosed cavity. The values of $C_{F b}\left(C_{F}\right.$ on the back surface) are obtained when $C_{D}{ }^{\prime}=0$ and the axial gaps of both cavities have the same size for different $\operatorname{Re}$ (under that condition $C_{F f}=C_{F b}$ ). After obtaining those values, the values of $C_{F f}$ with different values of $C_{D}{ }^{\prime}$ can be calculated with Equation (5).

$$
\begin{gathered}
F_{a}=F_{a b}-F_{a f} \\
F_{a f}=\pi \cdot p_{b} \cdot b^{2}-C_{F f} \cdot \rho \cdot \Omega^{2} \cdot b^{4} \\
F_{a b}=\pi \cdot p_{b} \cdot\left(b^{2}-a^{2}\right)-C_{F b} \cdot \rho \cdot \Omega^{2} \cdot\left(b^{4}-a^{4}\right) \\
C_{F f}=\frac{F_{a}+C_{F b} \cdot \rho \cdot \Omega^{2}\left(b^{4}-a^{4}\right)+\pi p_{b} a^{2}}{\rho \cdot \Omega^{2} \cdot b^{4}}
\end{gathered}
$$

\section{Test Rig Design and Experimental Set-Up}

The design of the test rig is shown in Figure 4. The cross section of the test rig is depicted in Figure $4 \mathrm{a}$. The centripetal through-flow (volumetric through-flow rate $Q$ ), shown by the black arrows in Figure 4a, is supplied with water by a pump system. The view along the " $A$ " direction is sketched in Figure $4 \mathrm{~b}$. The shaft sealing at the back cavity is depicted in Figure $4 \mathrm{c}\left(r_{\text {seal }}=10 \mathrm{~mm}\right)$. A picture of the test rig is shown in Figure $4 \mathrm{~d}$. The shaft is driven by an electric motor. A frequency converter is used to adjust the speed of rotation $(0 \sim 2500 / \mathrm{min})$ with the absolute uncertainty of $7.5 / \mathrm{min}$. In this study, only the axial gap of the front chamber is changed by installing six sleeves with different length. There are 24 channels in the guide vane (instead of entirely open at the periphery) to get more uniform centripetal through-flow, shown in Figure $4 \mathrm{~b}$. The area of each channel is $4 \times 10^{-6} \mathrm{~m}^{2}$. In this study, the channels in the guide vane are radial directed (see Figure $4 b$ ). Other parameters of the experiments in this study are given in Table 1 . The transducers in the test rig include two pressure transducers (36 pressure tubes), a torque transducer and three tension compression transducers. A thrust plate is fixed by a ball bearing and a nut from both sides to convey the axial thrust to the tension compression transducers. A linear bearing is used to minimize the frictional resistance during the axial thrust measurements. During the measurements of axial thrust, the calibrations of the axial thrust transducers are performed when changing the axial gap width of the front chamber. When measuring the torque, the values of the shaft without the disk rotating at different speeds of rotation are subtracted. The measured $R_{z}$ of the disk is $1 \mu \mathrm{m}$. The values of $R_{z}$ on all the other surfaces of the test rig are below $1.6 \mu \mathrm{m}$. 
(a) Cross section of the test rig

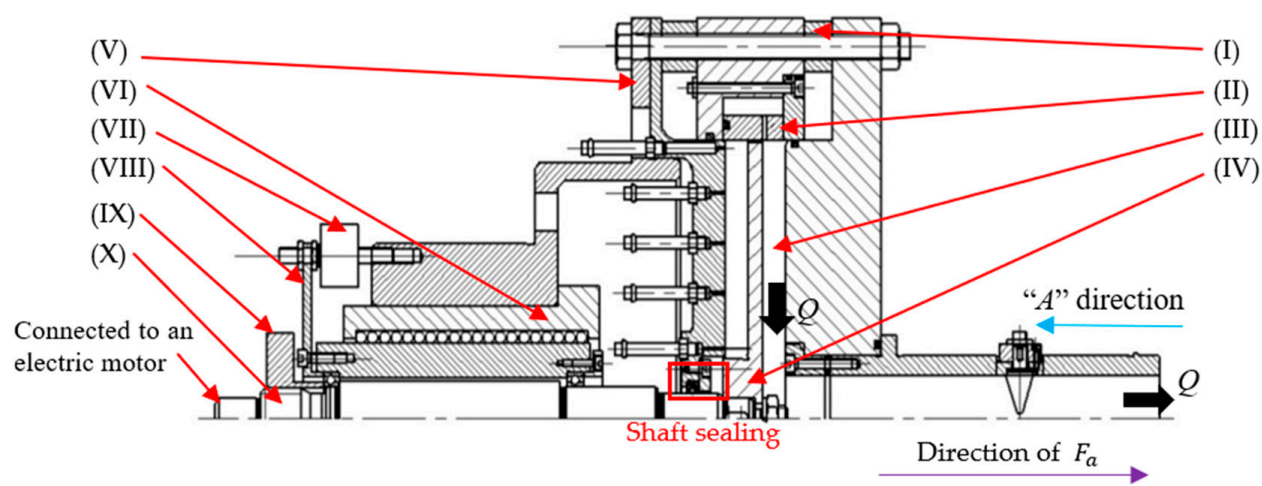

(I). Sleeves (to change the axial gap), (II). Guide vane (24 channels), (III). Front chamber, (IV). Disk, (V). Back cover, (VI). Linear bearing, (VII). Tension compression sensor, (VIII). Thrust plate, (IX). Nut, (X). Shaft

(b) View along " $\mathrm{A}$ " direction

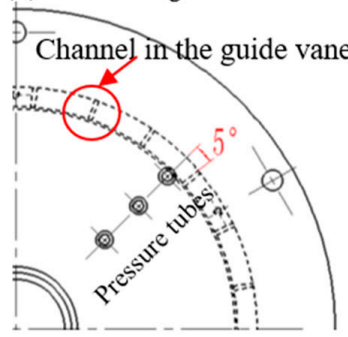

(c) Shaft sealing

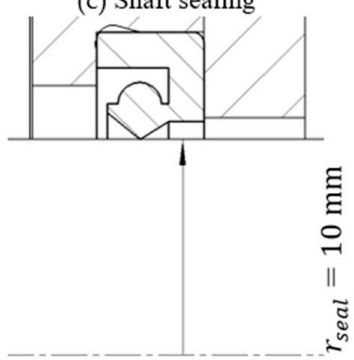

(d) Test rig

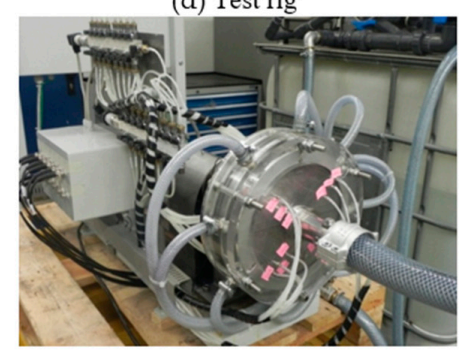

Figure 4. Test rig design. $F_{a}$ : axial thrust; $r_{\text {seal }}$ : radius of shaft seal.

Table 1. Parameters of the experiments.

\begin{tabular}{ccccccc}
\hline $\boldsymbol{b}(\mathbf{m m})$ & $n(/ \mathbf{m i n})$ & $Q\left(\mathbf{m}^{3} / \mathbf{s}\right)$ & $s(\mathbf{m m})$ & $s_{b}(\mathbf{m m})$ & $\boldsymbol{a}(\mathbf{m m})$ & $\boldsymbol{t}(\mathbf{m m})$ \\
\hline 110 & $0 \sim 2500$ & $-5.56 \times 10^{-4} \sim 0$ & $2 \sim 8$ & 8 & 23 & 10 \\
\hline \multicolumn{7}{c}{$n$ speed of rotation; $Q$ : volumetric through-flow rate. }
\end{tabular}

The measurements of the axial thrust include two steps. The cavities for each step are depicted in Figure 5. The first step is to measure the axial force imposed by the drive end of the motor when the shaft without the disk is rotating at different speed of rotation in the air. For the second step, all the results are modified by subtracting above values obtained at the first step according to the speed of rotation. The shaft head in Figure $5 b$ is considered as a part of front surface (with the area of $\Omega \cdot b^{2}$ ). The geometry of the nut is ignored. Then, the values of thrust coefficient on a single surface can be calculated with Equation (5).

(a) Step 1

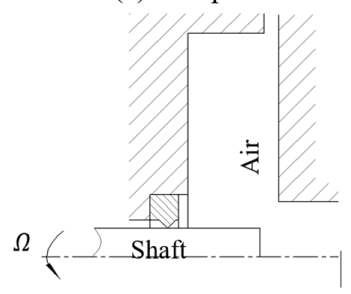

(b) Step 2

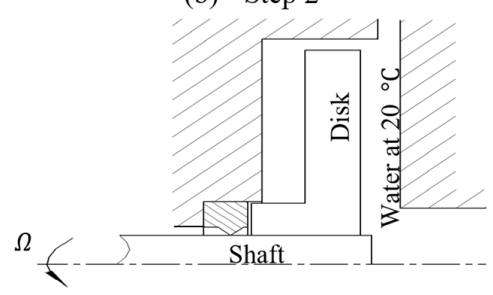

Figure 5. Cavities to measure the axial thrust. 
The relative error, noted as $\mathrm{e}_{T}$, of the pressure transducers is $1 \%$ (full scale (FS)). The value of $\mathrm{e}_{T}$ for the torque transducer is $0.1 \%(F S)$. The value of $\mathrm{e}_{T}$ for the axial thrust transducers is $0.5 \%$ (FS). All the experimental results are the ensemble average of 1000 samples. The uncertainties of the measured results, noted as $\Delta N$, are the differences between the real values and the measured values. They are estimated with the root sum squared method. The uncertainties are calculated with Equation (6). $N_{T}$ is the uncertainty due to the transducers. $N_{D}$ is the uncertainty due to the data acquisition system. The measuring range $\left(M_{r}\right)$ of the torque meter is $0 \sim 10 \mathrm{Nm}$. The measured range of the pressure transducer is $0 \sim 2.5$ bar (absolute pressure). The measured range of the thrust transducers is $-100 \sim 100 \mathrm{~N}$. The input voltage signals are the following ranges: $0 \sim 10 \mathrm{~V}$ for the pressure transducers and the torque transducer, $-10 \mathrm{~V} \sim 10 \mathrm{~V}$ for the axial thrust transducers. The absolute accuracy of the data acquisition system (with NI USB-6008) is $4.28 \mathrm{mV}$ in this study. The random noise and zero order uncertainty are neglected because they are very small. The distributions of the results are considered as normal distributions and the normal distribution coefficient is selected as 1.96 ( $95 \%$ confidence level). $n_{T}$ represents the number of the transducers used to obtain one result together. To evaluate $F_{a}$ and $M$ on the front surface, the total results, the results on the back surface (obtained when $C_{D}{ }^{\prime}=0$ and $s=s_{b}$ ) and the results when the shaft is rotating without disk are measured with the transducers. Hence, the measuring times to obtain one result, noted as $n_{M}$, is 3 for the axial thrust, the frictional torque $(M)$ and $\operatorname{Re}$ (by measuring $n$ ). The uncertainties of the measurements are summarized in Table 2.

$$
\Delta N=\sqrt{N_{T}^{2}+N_{D}^{2}} ; N_{T}=\frac{\sqrt{n_{T} \cdot n_{M} \cdot\left(e_{T} \cdot M_{r}\right)^{2}}}{1.96 \cdot \sqrt{1000}} ; N_{D}=\frac{\sqrt{n_{T} \cdot n_{M} \cdot\left(e_{D} \cdot M_{r}\right)^{2}}}{1.96 \cdot \sqrt{1000}}
$$

Table 2. Uncertainty analysis for the measurements.

\begin{tabular}{cccccc}
\hline & $\boldsymbol{p}$ (Bar) & $\boldsymbol{F}_{\boldsymbol{a}}(\mathbf{N})$ & $\boldsymbol{M} \mathbf{( N m )}$ & $\boldsymbol{R e}$ & $\boldsymbol{C}_{\boldsymbol{D}}{ }^{\prime}$ \\
\hline$\Delta \boldsymbol{N}$ & $4.04 \times 10^{-4}$ & $2.43 \times 10^{-2}$ & $3.00 \times 10^{-4}$ & $9.01 \times 10^{4}$ & 4.1 \\
$n_{\boldsymbol{T}}$ & 1 & 3 & 1 & 1 & 1 \\
$n_{\boldsymbol{M}}$ & 1 & 3 & 3 & 3 & 1 \\
\hline
\end{tabular}

$\Delta N$ : uncertainty of the measured results; $n_{T}$ : number of transducers; $n_{M}:$ measuring times to obtain one result; $p$ : pressure; $M$ : frictional torque; Re: global circumferential Reynolds number; $C_{D}{ }^{\prime}$ : through-flow coefficient.

\section{Numerical Simulation}

To predict the cavity flow, numerical simulations are carried out using the ANSYS CFX 14.0 code [15]. There are 24 channels in the guide vane. Considering the axial symmetry of the problem, a segment (15 degree) of the whole domain is modeled and a rotational periodic boundary condition is applied. Structured meshes are generated with ICEM 14.0. The domain for the numerical simulation when $G=0.072$ is depicted with yellow color in Figure 6. The simulation type is set as steady state. Barabas et al. [15] found that the simulation results from the shear stress transport (SST) $k-\omega$ turbulence model in combination with the scalable wall functions are in good agreement with the measured pressure in a rotor-stator cavity with air. The deviations of the pressure measurement are less than $1 \%$. Hence, in this study, the same turbulence model and wall functions are used. The boundary conditions at the inlet and at the outlet are pressure inlet and mass flow outlet, respectively. The values of the pressure at the inlet are set according to the pressure sensor at the pump outlet. The convergence criteria for all the numerical simulations are set as $10^{-5}$ in maximum type. The turbulent numeric is set as second order upwind. The maximum value of $y^{+}$in all the simulation models is 13.4. 


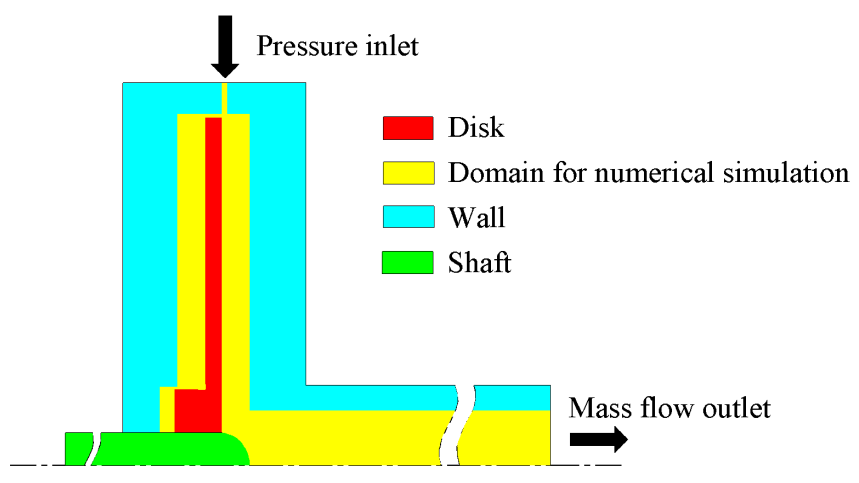

Figure 6. Domain for numerical simulation when $G=0.072$.

\section{Results and Discussion}

\subsection{Simulation Results of Velocity Distributions}

The velocity profiles are sensitive to the boundary condition at the inlet. The profiles in the front chamber are discussed in this part because there is a small jet flow through each channel in the guide vane. All the velocities are made dimensionless by dividing them by $\Omega \cdot b$. The values of $V_{z}$ are positive when they have a direction from the disk to the wall. The velocity profiles at three radial positions for $R e=1.9 \times 10^{6}$ and $G=0.072$ (wide gap) are shown in Figure 7. The dimensionless radial velocities are not exactly zero in the central cores, shown in Figure $7 \mathrm{a}-\mathrm{c}$. From the distribution of tangential velocity, there are central cores at all the investigated radial positions where the values of $V_{\varphi}$ are almost constant, shown in Figure $7 \mathrm{~d}-\mathrm{f}$. At $x=0.955$ and $x=0.79$, the values of the tangential velocity are smaller at $\zeta=0.5$ when $\left|C_{D}^{\prime}\right|$ increase from 1262 to 3787 and 5050, depicted in Figure 7d,e. The profiles are special, because according to the former results (such as from Poncet et al. [9] and Debuchy et al. [11]), the increase of $\left|C_{D}^{\prime}\right|$ will result in an increase of the core swirl ratio $K$ for centripetal through-flow. Then, the tangential velocity should increase instead of decrease. Probably this can be attributed to the jet flow through the channel at the inlet, which is stronger at large $\left|C_{D}{ }^{\prime}\right|$. At $x=0.955$, the values of $\left|V_{z}\right|$ become smaller for larger $\left|C_{D}^{\prime}\right|$ in general, shown in Figure $7 \mathrm{~g}$. The direction of $V_{z}$ is from the disk towards the wall for $\left|C_{D}^{\prime}\right|=0$ and $\left|C_{D}^{\prime}\right|=1262$ at $x=0.955$, while it is from the wall towards the disk at $\left|C_{D}^{\prime}\right|=3787$ and $\left|C_{D}^{\prime}\right|=5050$. There are axial circulations of the fluid in the front chamber. The directions of the axial circulations, however, are strongly influenced by the values of $\left|C_{D}{ }^{\prime}\right|$, depicted in Figure $7 \mathrm{~g}-\mathrm{i}$.

The velocity profiles at the three radial coordinates for $R e=1.9 \times 10^{6}$ and $G=0.018$ (small gap) are shown in Figure 8. The dimensionless radial velocities $V_{r}$ vary along $\zeta$, shown in Figure 8a-c. The values of $V_{r}$ decrease with the increase of $\left|C_{D}^{\prime}\right|$ in general. The tangential velocity $V_{\varphi}$ decreases constantly with the increase of $\zeta$, which is the characteristic of the regime III, shown in Figure 8d-f. At $x=0.955$ and $x=0.79$, the values of tangential velocity are much smaller at large $\left|C_{D}{ }^{\prime}\right|$, shown in Figure $8 \mathrm{~d}$. The reason is that the impact of the jet flow at the inlet becomes greater for smaller $G$. The profiles of $V_{z}$ are quite different at $x=0.955$ in Figure $8 \mathrm{~g}$, compared with those in Figure $7 \mathrm{~g}$. The values of $V_{z}$ are less than those from Figure $7 \mathrm{~h}, \mathrm{i}$. The results of $V_{z}$ indicate that the axial circulations of fluid are sensitive to the values of $G$. 


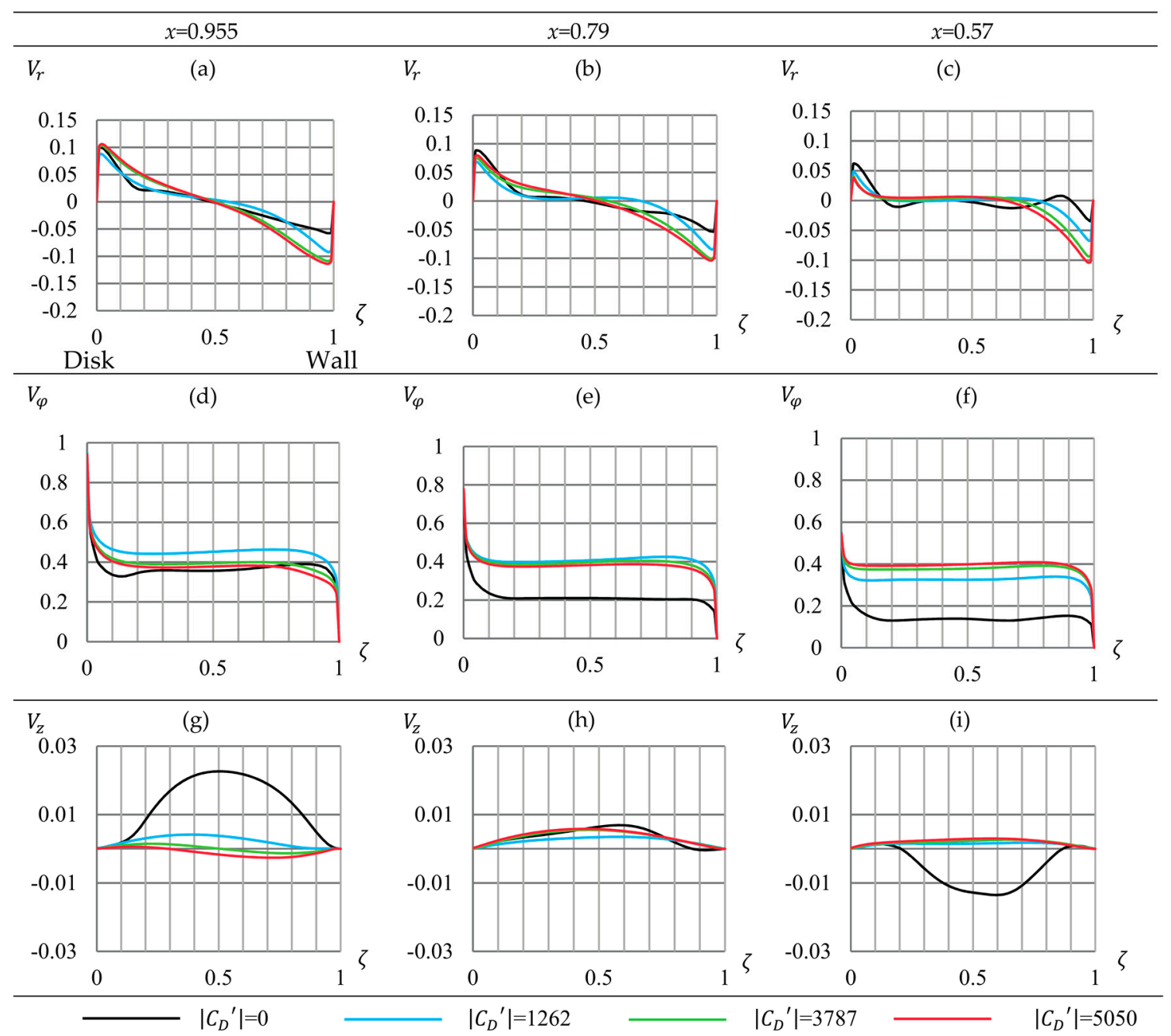

Figure 7. Velocity profiles for $R e=1.9 \times 10^{6}$ and $G=0.072$ (wide gap). G: dimensionless axial gap. 

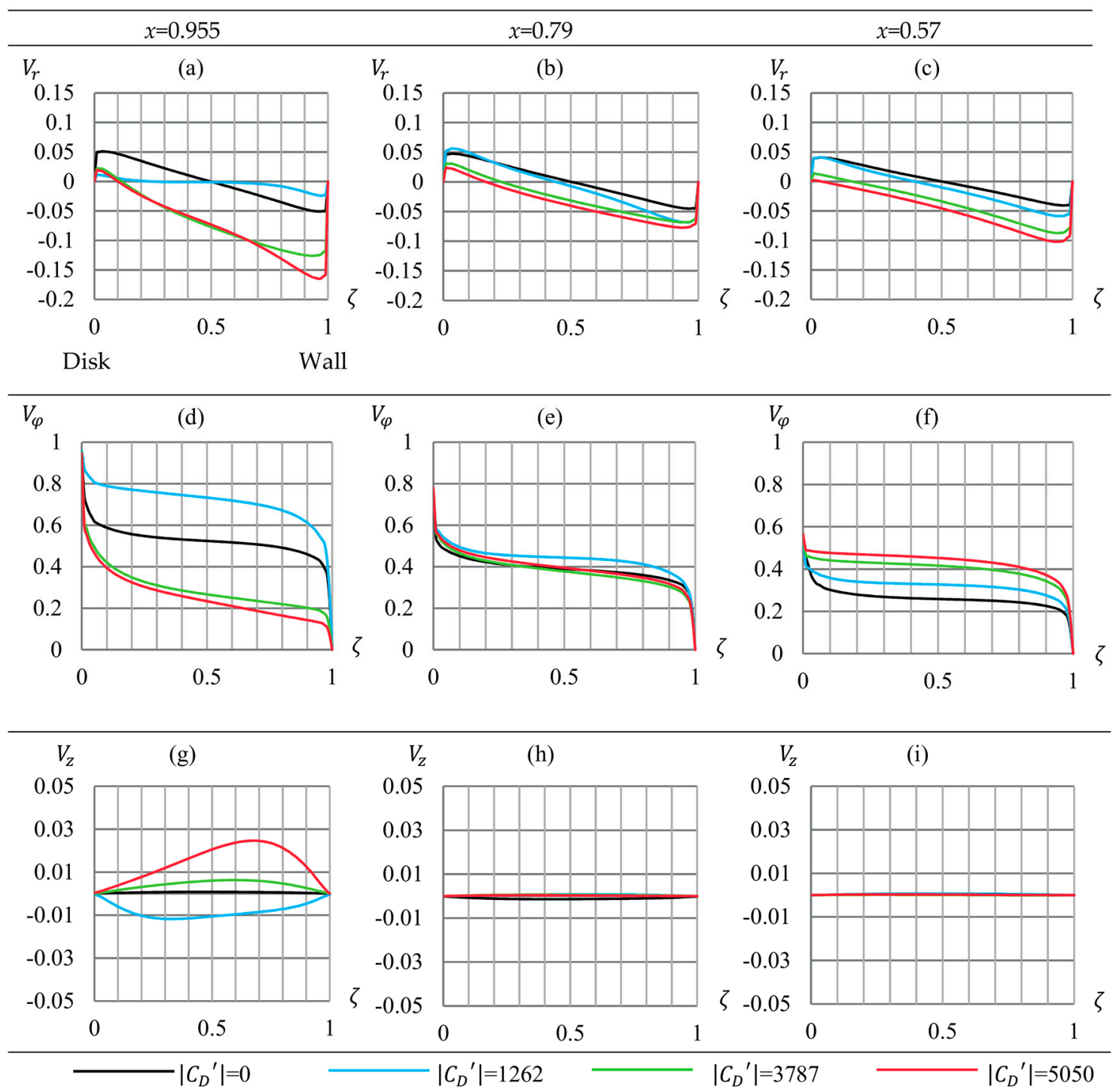

Figure 8. Velocity profiles for $R e=1.9 \times 10^{6}$ and $G=0.018$ (small gap).

\subsection{Pressure Distributions}

Due to the construction of the geometry, there is no pressure tube at $x=1$. The closest tube is at $x=0.955$. The pressure values at $x=1$ (reference pressure) are taken from numerical simulation. Since the values of $p$ from numerical simulation at $x=0.955$ are close to those from experiments, the small errors are neglected.

The pressure coefficient $C_{p}$ are positive values because the pressure drops towards the shaft. In Figure 9, the values of $C_{p}$ are plotted versus the dimensionless radial coordinates for three values of $R e$ and $G$. The experimental results show that the values of $C_{p}$ increase with increasing $\left|C_{D}{ }^{\prime}\right|$. The values of $C_{p}$ decrease with the increase of $R e$ and $G$ in general. When $R e=1.9 \times 10^{6}$ and $R e=2.79 \times 10^{6}$, the uncertainties of the $C_{p}$ are respectively $2.7 \times 10^{-4}$ and $1.3 \times 10^{-4}$, which are very small compared with the measured results. Hence, they are neglected in Figure $9 \mathrm{~d}-\mathrm{i}$. 

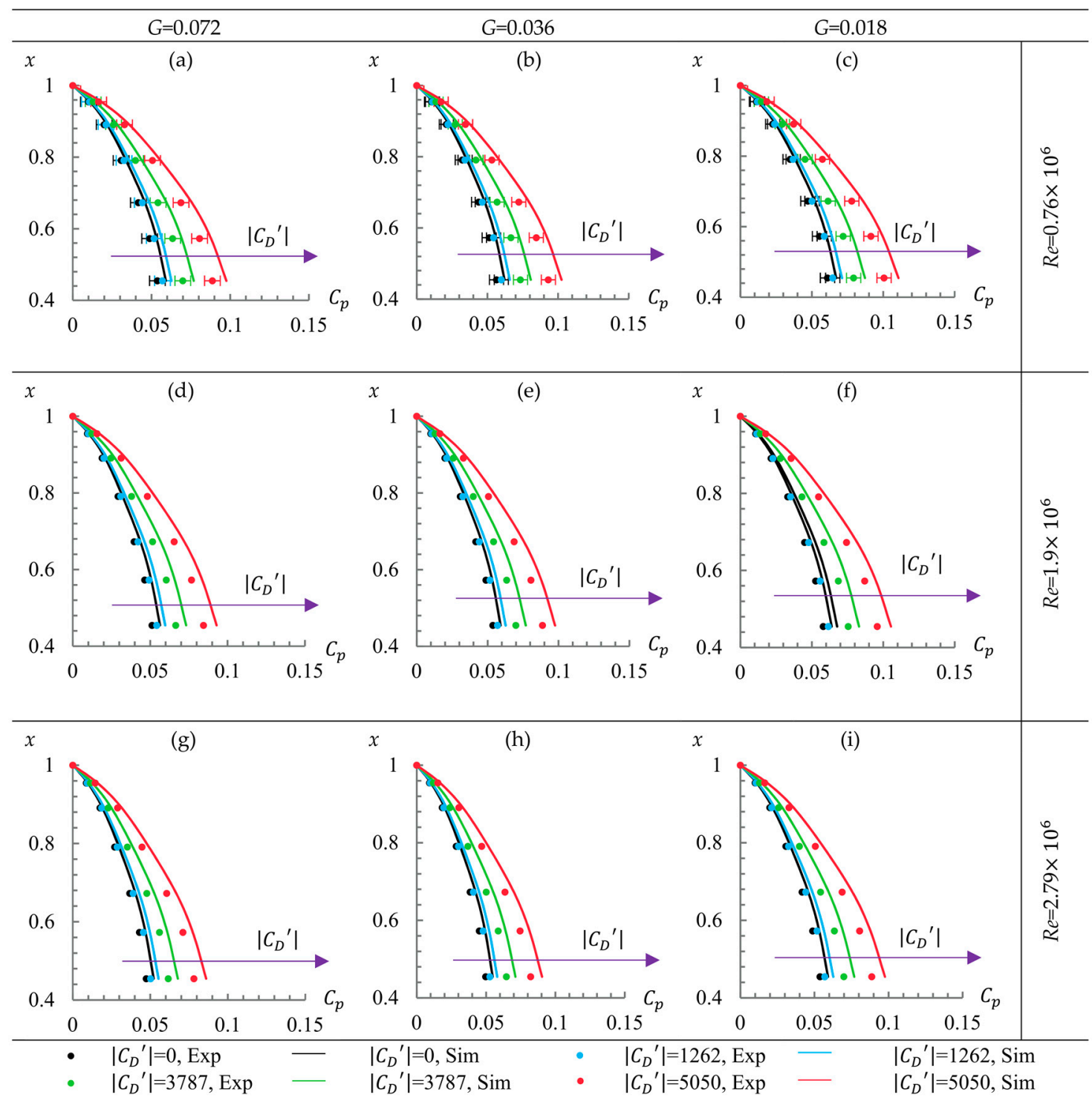

Figure 9. Influence of $C_{D}{ }^{\prime}$ on $C_{p}$ (pressure coefficient) in dependence of $R e$ and $G$.

\subsection{Axial Thrust Coefficient}

Based on the axial thrust measurements (direction of the axial thrust see Figure 4a), a correlation for $C_{F f}$ is determined, given in Equation (7). The results from Equation (7) are consistent with the experimental results, plotted in Figure 10. With the increase of $\left|C_{D}{ }^{\prime}\right|$, the values of $C_{F f}$ increase, which can be attributed to the drop of $p$. The values of $C_{F f}$ are smaller for higher values of $G$ and $R e$.

$$
C_{F f}=\left[6.6 \cdot 10^{-3} \cdot \ln (R e)-0.113\right] \cdot e^{\left(1.2 \cdot 10^{-4} \cdot\left|C_{D}\right|\right)} \cdot[0.122 \cdot \ln (G)-0.67]
$$



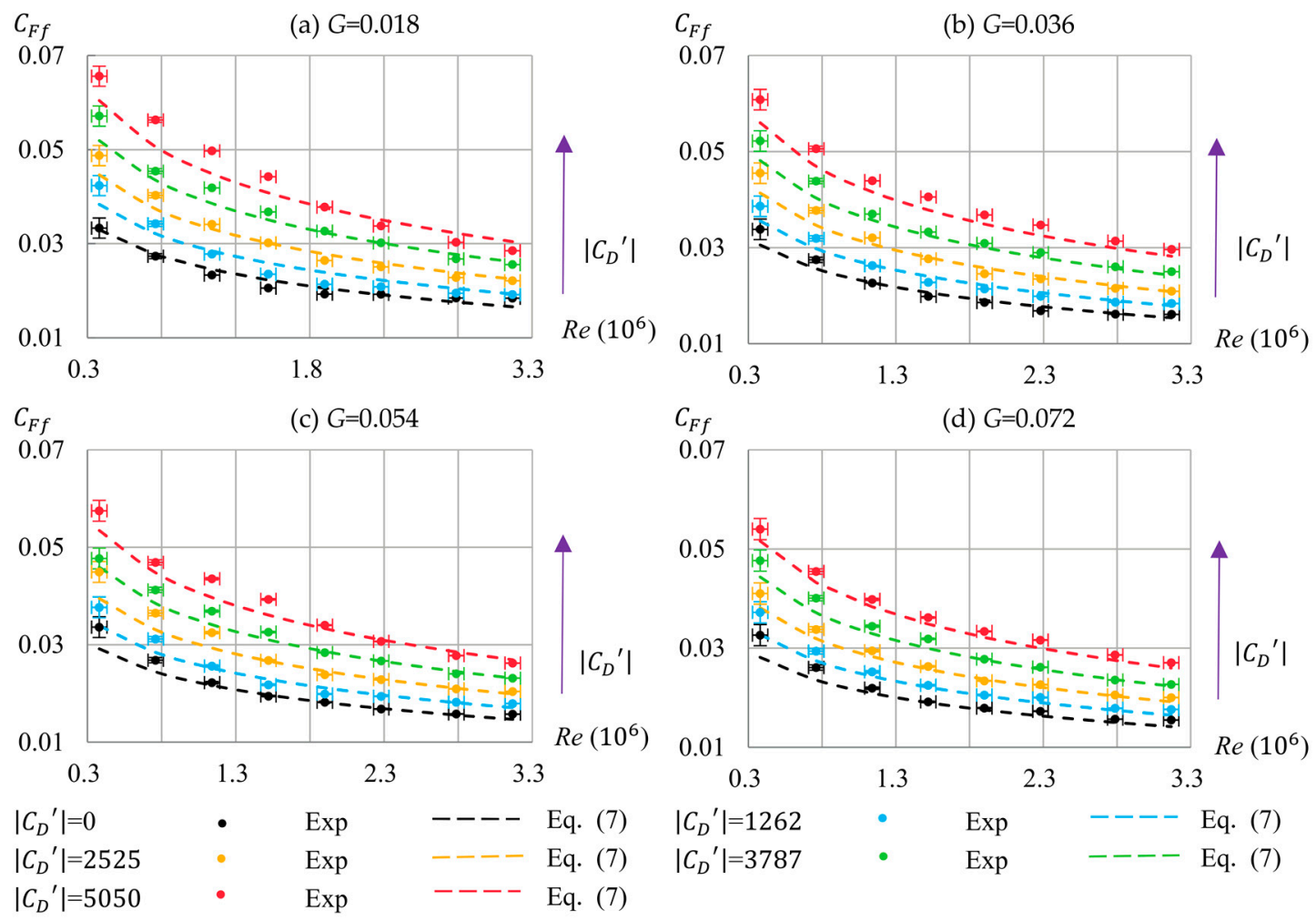

Figure 10. Mean $C_{F f}-C_{D}{ }^{\prime}$ curves in dependence of Re and G. $C_{F}$ : axial thrust coefficient; $C_{F f}: C_{F}$ on the front surface.

\subsection{The 3D Daily and Nece Diagram}

The typical tangential velocity profiles for regime III (merged disk boundary layer and wall boundary layer) and regime IV (separated disk boundary layer and wall boundary layer) are given in Figure 11.
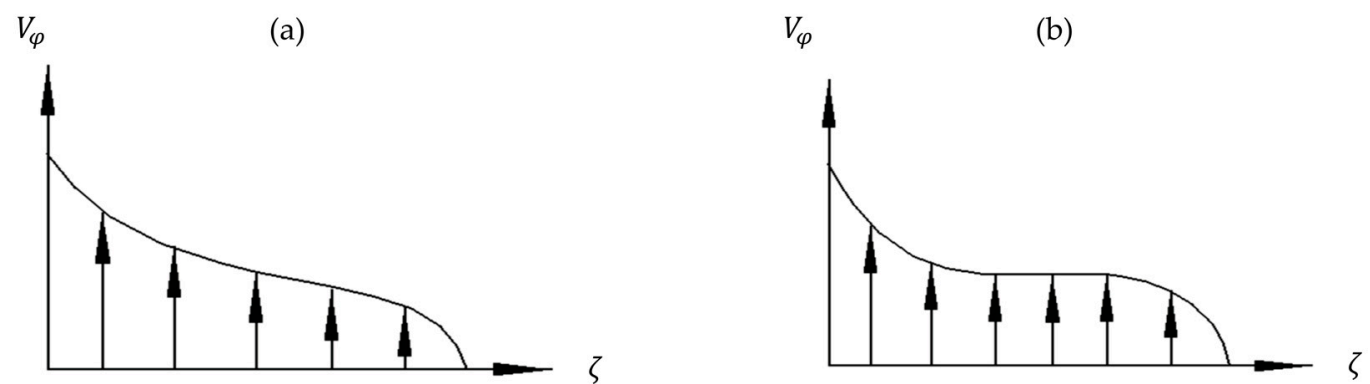

Figure 11. Typical tangential velocity profiles for: (a) regime III and (b) regime IV.

Based on the simulation results of the tangential velocity, part of the Daily and Nece diagram (see Figure 1) is extended into 3D by distinguishing the tangential velocity profiles (see Figure 11) at $x=0.955, x=0.79$ and $x=0.57$. The scope of this study is the following parameter ranges: $\left|C_{D}{ }^{\prime}\right| \leq 5050$, $0.38 \times 10^{6} \leq \operatorname{Re} \leq 3.17 \times 10^{7}$ and $0.018 \leq G \leq 0.072$. They are categorized into two regimes, namely regime III (below the distinguishing lines) and regime IV (above the distinguishing lines). Currently, five distinguishing lines are found for different $\left|C_{D}{ }^{\prime}\right|$ shown in Figure 12b. The distinguishing line at $C_{D}{ }^{\prime}=0$ is almost equal to that from Daily and Nece [3]. The distinguishing lines become steeper for higher values of $\left|C_{D}{ }^{\prime}\right|$. The approximate distinguishing surface is drawn through the lines, shown in Figure 12a. Below and above the surface are regime III and regime IV, respectively. Near the 
distinguishing surface, there is a mixing zone where regime III and regime IV coexist in the front chamber. In this study, it is not plotted in Figure 12.
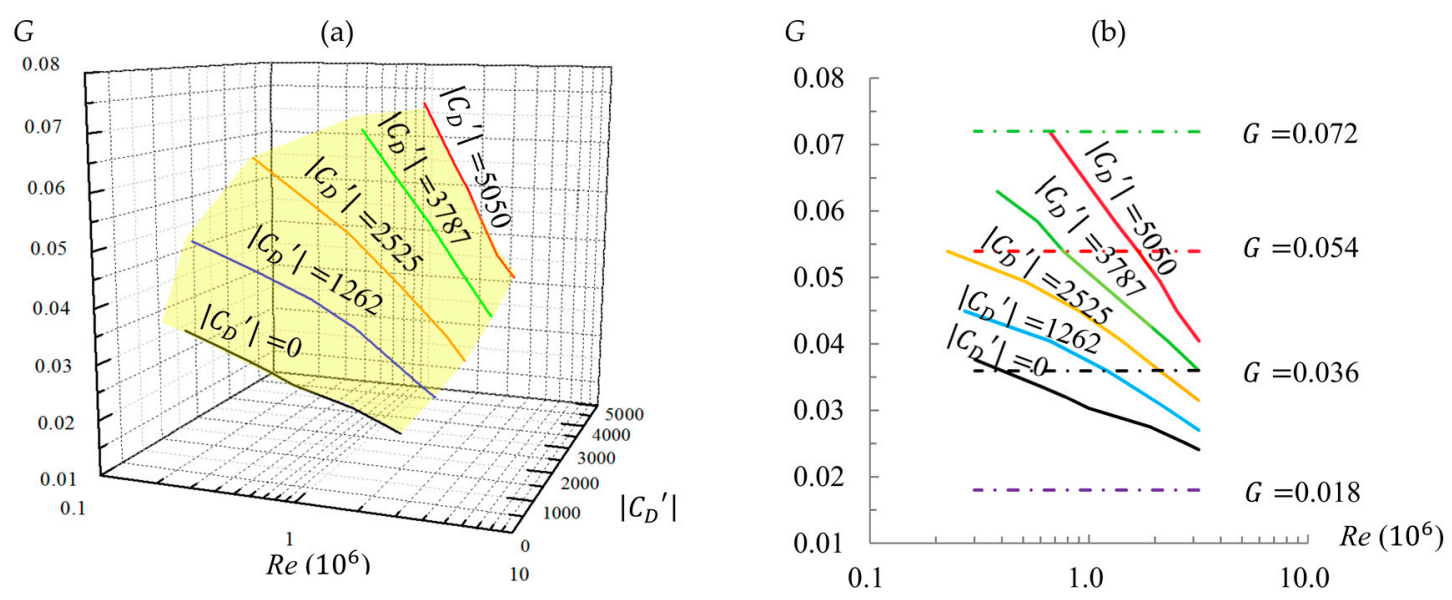

Figure 12. Part of the 3D Daily and Nece diagram: (a) Distinguishing surface and (b) Distinguishing lines.

\subsection{Moment Coefficient}

According to the experimental results from Han et al. [19], the moment coefficient on the cylinder surface of the disk $\left(C_{M c y l}\right)$ can be estimated with Equation (8) for smooth disks.

$$
C_{\text {Mcyl }}=\frac{2 \cdot\left|M_{c y l}\right|}{\rho \cdot \Omega^{2} \cdot b^{5}}=\frac{0.084 \cdot \pi \cdot t}{b \cdot\left(\lg \frac{\Omega \cdot b^{2}}{v}\right)^{1.5152}}
$$

When $C_{D}{ }^{\prime}=0$, the values of $C_{M}$ for $G=0.018$ (regime III) and $G=0.072$ (regime IV) are compared in Figure 13a,b, respectively. The differences between the experimental results and those from the correlations by Daily and Nece [3] for both regime III and regime IV, are colossal. $k_{s}$ is the equivalent surface roughness, defined in Equation (11). According to Schlichting et al. [7], $k_{s l}$ (the limitation for hydraulic smooth) wall can be estimated with Equation (12). The disk therefore can be considered hydraulic smooth when $R_{z} \leq 38.2 \mu \mathrm{m}$. To explain the wide gap, the results for a rougher disk $\left(R_{z}=22 \mu \mathrm{m}\right)$ are also plotted for comparison. The results are much closer to those from the equation by Daily and Nece [3]. Hence, the differences can be attributed to the difference of surface roughness. Equations (9) and (10) are therefore determined to satisfy the experimental results $\left(R_{z}=1 \mu \mathrm{m}\right.$ for the disk).

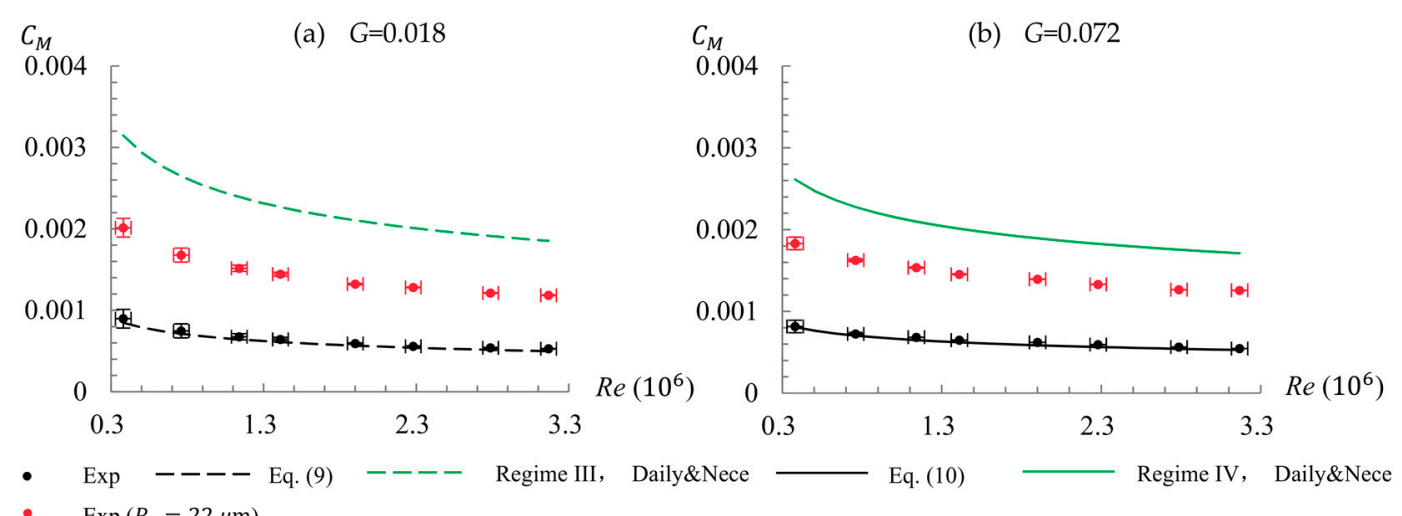

Figure 13. Comparison of the results of $C_{M}$ (moment coefficient) for $G=0.018$ and $G=0.072$ at $C_{D}{ }^{\prime}=0$. 


$$
\begin{gathered}
C_{M 3}=0.011 \cdot G^{-\frac{1}{6}} \cdot R e^{-\frac{1}{4}} \cdot\left[e^{\left(0.8 \cdot 10^{-4} \cdot\left|C_{D}{ }^{\prime}\right|\right)}\right] \\
C_{M 4}=0.014 \cdot G^{\frac{1}{10}} \cdot \operatorname{Re}^{-\frac{1}{5}} \cdot\left[e^{\left(0.46 \cdot 10^{-4} \cdot\left|C_{D}{ }^{\prime}\right|\right)}\right] \\
k_{s}=\frac{\pi \cdot \varepsilon}{8} \cdot \varepsilon=0.978 \cdot R_{z} \\
k_{s l}=\frac{100 \cdot v}{(1-K) \cdot r \cdot \Omega}
\end{gathered}
$$

To introduce the influence of $C_{D}{ }^{\prime}$ on the moment coefficient, the results of $C_{M}$ from both the experiments and the equations are plotted versus $R e$ in Figure 14 . With the increase of $R e$, the flow regime may change from regime III to regime IV (see the distinguishing lines in Figure 12). For $G=0.018$ and $G=0.036$, most of the flow regimes are regime III and the results are close to those from Equation (9) in general, shown in Figure 14a,b. The flow regimes change from regime III to regime IV with the increase of $\operatorname{Re}$ for $\left|C_{D}{ }^{\prime}\right|=0$ and $\left|C_{D}{ }^{\prime}\right|=1262$ at $G=0.036$ in Figure $14 \mathrm{~b}$. For $G=0.054$ and $G=0.072$, most of the flow regimes are regime IV and the results are close to those from Equation (10) in general, depicted in Figure 14c,d. The results of $C_{M}$ from the equations are in good agreement with those from experiments. The values of $C_{M}$ increase with the increase of $\left|C_{D}{ }^{\prime}\right|$, while decrease with the increase of $R e$. At large values of $R e$, the impact of $C_{D}{ }^{\prime}$ on $C_{M}$ becomes lesser. At the same values of $\left|C_{D}{ }^{\prime}\right|$, the intersection points of the curves from Equation (9) and those from Equation (10) are close to those in Figure 12b. The difference can be attributed to the existence of the mixing zone.
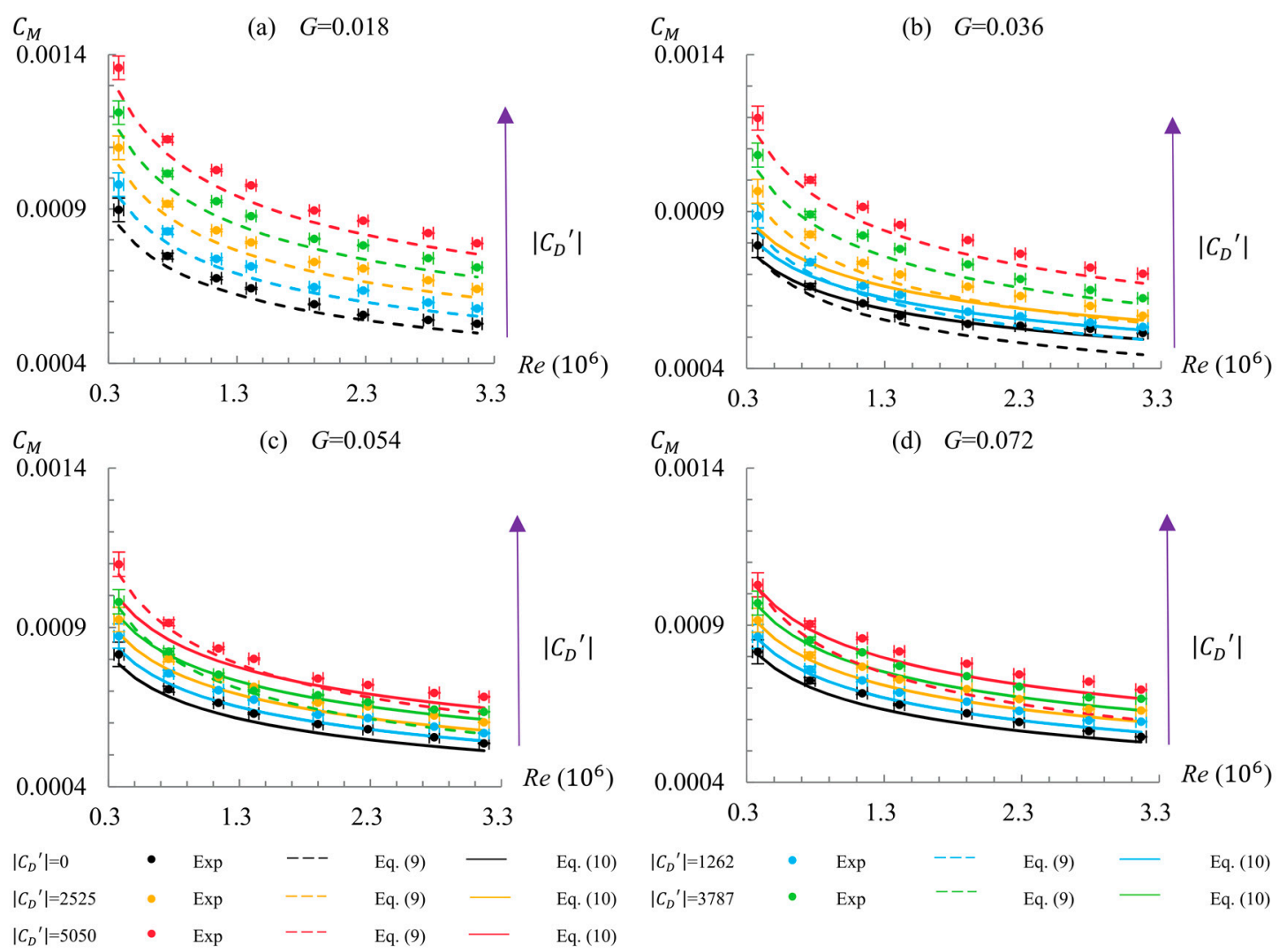

Figure 14. Curves for $C_{M}$ in dependence of $C_{D}{ }^{\prime}$ for different values of $R e$ and $G$.

The results from Equations (9) and (10) at the distinguishing lines should be equal. The results of $C_{M 3} / C_{M 4}\left(C_{M}\right.$ for regime III/ $C_{M}$ for regime IV) for a non-dimensioned gap width $G$ at the distinguishing lines (see Figure 12b) are presented in Figure 15. The differences, attributed to the 
existence of the mixing zone, are less than $4 \%$. The results indicate that the distinguishing lines (in Figure 12), Equations (9) and (10) are reasonable.

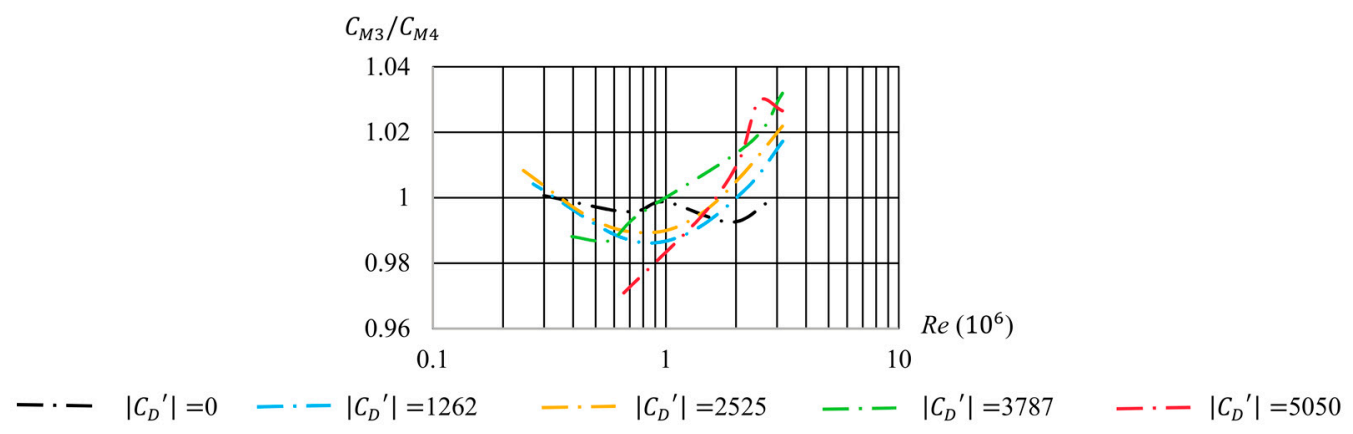

Figure 15. Results of $C_{M 3} / C_{M 4}\left(C_{M}\right.$ for regime III/ $C_{M}$ for regime IV) at the distinguishing lines.

An example is provided on the applications of the results in this paper. Shi et al. [20] studied the axial thrust of a single stage well pump based on both numerical simulation and experiments. The pressure acting at the impeller is shown in Figure 16a. Based on Equation (7), the thrust coefficient at surface 1 and surface 2 can be calculated when the leakage flow is estimated. The volumetric leakage through-flow rate is considered as $5 \%$ of the flow rate of the pump. The force at the impeller eye (at surface 3) with numerical simulation. Then, the axial thrust of the impeller can be calculated when the axial force of the shaft is estimated. They predicted the force on all the surfaces of the impeller and the shaft to calculate the axial thrust. The axial force of the shaft is obtained at different flow rate from the simulation results by Shi et al. [20]. The maximum difference between their simulation results and measurements of the axial thrust is $5.9 \%$. The values of $F_{a b}-F_{a f}$ are plotted versus $C_{D}{ }^{\prime}$ in Figure 16b. The experimental results of $F_{a b}-F_{a f}$ are obtained by subtracting the forces on the rest of the surfaces (from numerical simulation). The results from Equation (7) are in better agreement with the experimental results than those from the equation by Kurokawa et al. [4] when $C_{D}{ }^{\prime}$ ranges from 1150 to 4630 .

(a)

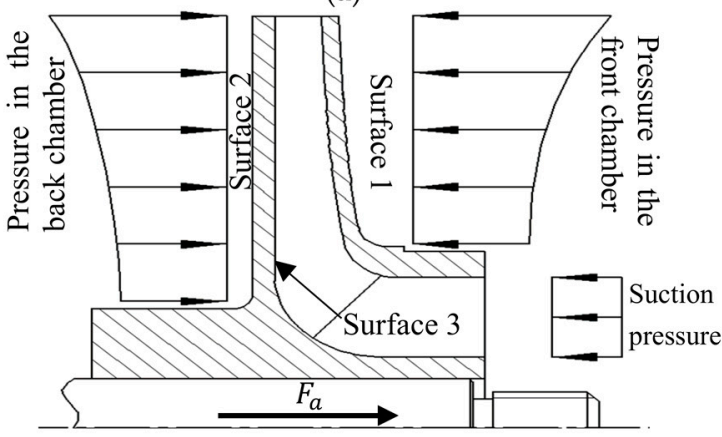

(b)

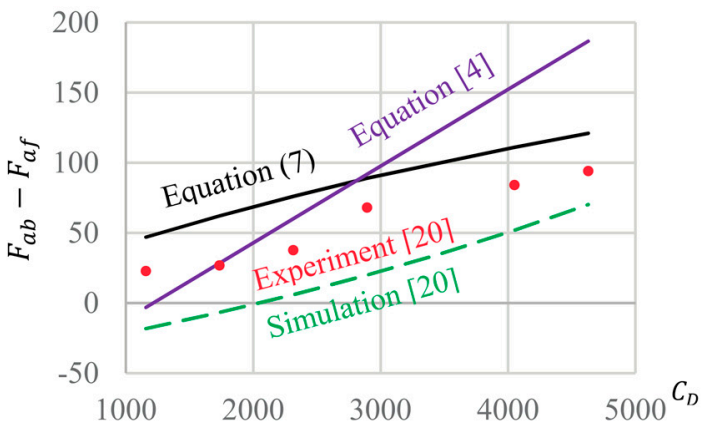

Figure 16. Axial thrust in a centrifugal single stage well pump [20]: (a) Pressure distribution and (b) Comparison of $F_{a b}-F_{a f}$ [20]. $F_{a b}$ : force on the back surface of the disk; $F_{a f}$ : force on the front surface of the disk.

There are still some limitations of this work. The results and correlations are limited to non-preswirl centripetal through-flow. The centripetal leakage flow in the radial pumps and turbines, however, contains a certain amount of angular momentum, which deserves further investigation. All the experimental results are obtained with the smooth disk $\left(R_{z}=1 \mu \mathrm{m}\right)$. The applications of the equations are still limited because all of the results are influenced by the surface roughness of the disks. Some more 
results will be presented with rough disks and the equations will be modified by introducing the impact of the surface roughness of the disks in the next step. Currently, the distinguishing lines for regime III and regime IV are obtained by evaluating the tangential flow component based on numerical simulation. This will be put on an experimental level by measuring the velocity components in both tangential and radial direction with a Laser Doppler Velocimetry (LDV) system in the future.

\section{Conclusions}

The influence of centripetal through-flow on the velocity, radial pressure distribution, axial thrust and frictional torque in a rotor-stator cavity with different axial gaps is illustrated to be strong.

A correlation is determined, which enables to predict the influence of $G, \operatorname{Re}$ and $C_{D}{ }^{\prime}$ on the thrust coefficient $C_{F}$ for a smooth disk $\left(R_{z}=1 \mu \mathrm{m}\right)$.

For the first time, part of the 3D Daily and Nece diagram is obtained by distinguishing the tangential velocity profiles. Currently, the flow regimes are catagorized into two regimes, namely regime III and regime IV. Five distinguishing lines and the approximate distinguishing surface are presented. Two correlations are determined to predict the influence of $C_{D}{ }^{\prime}$ on $C_{M}$ for the two regimes with good accuracy for the smooth disk $\left(R_{z}=1 \mu \mathrm{m}\right)$. At the distinguishing lines, the results from the two equations are very close.

Using the equations for the axial thrust coefficient and the moment coefficient, the influence of the centripetal through-flow can be better considered when designing radial pumps and turbines with smooth impellers. Some more attention will be drawn in the future to the impact of the disk roughness. The 3D Daily and Nece diagram will be modified based on the velocity measurements with a LDV system.

Acknowledgments: This study is funded by China Scholarship Council and the chair of turbomachinery at University of Duisburg-Essen.

Author Contributions: Bo Hu, Dieter Brillert, Hans Josef Dohmen and Friedrich-Karl Benra devised and designed the experiments; Hans Josef Dohmen guided the design and the construction of the test rig; Dieter Brillert was responsible for the lab safety and the risk assessment; Friedrich-Karl Benra contributed the funds for the experiments and was supervisor during the research; Bo Hu did all the measurements and wrote the paper while Friedrich-Karl Benra reviewed the paper.

Conflicts of Interest: The authors declare no conflict of interest.

\section{Nomenclature}

\section{Latin Symbols}

a Hub radius

$b \quad$ Outer radius of the disk

$C_{D}{ }^{\prime} \quad$ Through-flow coefficient

$C_{F} \quad$ Axial thrust coefficient

$C_{F f} \quad C_{F}$ on the front surface

$C_{F b} \quad C_{F}$ on the back surface

$C_{M} \quad$ Moment coefficient

$C_{\text {Mcyl }}$ Moment coefficient on the cylinder surface of the disk

$C_{M 3} \quad C_{M}$ for regime III

$C_{M 4} \quad C_{M}$ for regime IV

$C_{p} \quad$ Pressure coefficient

$C_{q r} \quad$ Local flow rate coefficient

$\mathrm{e}_{T} \quad$ Relative error of the transducer

$\mathrm{e}_{D} \quad$ Relative error due to the data acquisition device

$F_{a} \quad$ Axial thrust

$F_{a f} \quad$ Force on the front surface of the disk

$F_{a b} \quad$ Force on the back surface of the disk 
G Dimensionless axial gap

$K \quad$ Core swirl ratio at $\zeta=0.5$

$k_{s} \quad$ Equivalent surface roughness

$k_{s l} \quad$ Limitation of $k_{s}$ for hydraulic smooth wall

$M \quad$ Frictional torque

$M_{c y l} \quad$ Frictional resistance on the cylinder surface of the disk

$M_{r} \quad$ Measured range

$\dot{m} \quad$ Mass flow rate

$N_{D} \quad$ Uncertainty of the data acquisition system

$N_{T} \quad$ Uncertainty of the transducer

$\Delta N \quad$ Uncertainty of the measured results

$n \quad$ Speed of rotation

$n_{T} \quad$ Number of transducers

$n_{M} \quad$ Measuring times to obtain one result

$p \quad$ Pressure

$p_{b} \quad$ Pressure at $r=b$

$p^{*} \quad$ Dimensionless pressure

$Q \quad$ Volumetric through-flow rate

$\mathrm{Re} \quad$ Global circumferential Reynolds number

$R_{\varphi} \quad$ Local circumferential Reynolds number

$r \quad$ Radial coordinate

$r_{\text {seal }} \quad$ Radius of shaft seal

$s \quad$ Axial gap of the front chamber

$s_{b} \quad$ Axial gap of the back chamber

$t \quad$ Thickness of the disk

$V_{r} \quad$ Dimensionless radial velocity

$V_{z} \quad$ Dimensionless axial velocity

$V_{\varphi} \quad$ Dimensionless tangential velocity

$x \quad$ Dimensionless radial coordinate

$z \quad$ Axial coordinate

\section{Greek Symbols}

$\varepsilon \quad$ Diameter of spheres

$\zeta \quad$ Dimensionless axial coordinate

$\mu \quad$ Dynamic viscosity of water

$v \quad$ Kinematic viscosity of water

$\rho \quad$ Density of water

$\Omega \quad$ Angular velocity of the disk

\section{Abbreviations}

FS Full scale

LDA Laser Doppler Anemometer

LDV Laser Doppler Velocimetry

SST Shear Stress Transport

\section{References}

1. Kármán, T.V. Über laminare und turbulente Reibung. Z. Angew. Math. Mech. 1921, 1, 233-252. [CrossRef]

2. Cochran, W.G. The flow due to a rotating disk. Proc. Camb. Philos. Soc. 1934, 30, 365-375. [CrossRef]

3. Daily, J.W.; Nece, R.E. Chamber dimension effects on induced flow and frictional resistance of enclosed rotating disks. J. Basic Eng. 1960, 82, 217-232. [CrossRef]

4. Kurokawa, J.; Toyokura, T. Study on axial thrust of radial flow turbomachinery. In Proceedings of the 2nd International JSME Symposium Fluid Machinery and Fluid Mechanics, Tokyo, Japan, September 1972; pp. 31-40. 
5. Kurokawa, J.; Toyokura, T. Axial Thrust, Disc Friction Torque and Leakage Loss of Radial Flow Turbomachinery. In Proceedings of the International Conference on Pump and Turbine Design and Development, Glasgow, UK, 1-3 September 1976.

6. Kurokawa, J.; Toyokura, T. Roughness Effects on the Flow along an Enclosed Rotating Disc. Bull. JSME 1978, 21, 1725-1732. [CrossRef]

7. Schlichting, H.; Gersten, K. Grenzschicht-Theorie; Springer: Berlin, Germany, 2006.

8. Goldstein, S. On the resistance to the rotation of a disc immersed in a fluid. Proc. Camb. Philos. Soc. 1935, 31, 232-241. [CrossRef]

9. Poncet, S.; Chauve, M.P.; Le Gal, P. Turbulent rotating disk flow with inward throughflow. J. Fluid Mech. 2005, 522, 253-262. [CrossRef]

10. Batchelor, G.K. Note on a class of solutions of the Navier-Stokes equations representing steady rotationallysymmetric flow. Q. J. Mech. Appl. Math. 1951, 4, 29-41. [CrossRef]

11. Debuchy, R.; Abdel Nour, F.; Bois, G. On the flow behavior in rotor-stator system with superimposed flow. Int. J. Rotating Mach. 2008, 2008, 719510. [CrossRef]

12. Launder, B.; Poncet, S.; Serre, E. Laminar, Transitional, and Turbulent Flows in Rotor-Stator cavities. Annu. Rev. Fluid Mech. 2010, 42, 229-248. [CrossRef]

13. Coren, D.; Childs, P.R.N.; Long, C.A. Windage sources in smooth-walled rotating disc systems. Proc. Inst. Mech. Eng. Part C 2009, 223, 873-888. [CrossRef]

14. Long, C.A.; Miles, A.L.; Coren, D.D. Windage Measurements in a Rotor Stator Cavity with Rotor Mounted Protrusions and Bolts. In Proceedings of the ASME Turbo Expo 2012: Turbine Technical Conference and Exposition, Copenhagen, Denmark, 11-15 June 2012.

15. Barabas, B.; Clauss, S.; Schuster, S.; Benra, F.-K.; Dohmen, H.J. Experimental and numerical determination of pressure and velocity distribution inside a rotor-stator cavity at very high circumferential Reynolds numbers. In Proceedings of the 11th European Conference on Turbomachinery, Madrid, Spain, 23-27 March 2015.

16. Will, B.C.; Benra, F.K. Investigation of the Fluid Flow in a Rotor-Stator Cavity with Inward Through-Flow. In Proceedings of the FEDSM2009, ASME Fluids Engineering Conference, Vail, CO, USA, 2-6 August 2009.

17. Will, B.C.; Benra, F.-K.; Dohmen, H.J. Numerical and Experimental Investigation of the Flow in the Side Cavities of a Centrifugal Pump. In Proceedings of the 12th International Symposium on Transport Phenomena and Dynamics of Rotating Machinery, Honolulu, HI, USA, 4-7 April 2010.

18. Will, B.C.; Benra, F.-K.; Dohmen, H.J. Investigation of the Flow in the Side Chambers of a Centrifugal Pump with Volute Casing. In Proceedings of the 10th International Symposium on Experimental Computational Aerothermodynamics of Internal Flows, Brussels, Belgium, 4-7 July 2011.

19. Han, H.; Gao, S.; Li, J.; Zhang, Y. Exploring fluid resistance of disk rotor based on boundary layer theory. Mech. Sci. Technol. Aerosp. Eng. 2015, 34, 1621-1625.

20. Shi, W.-D.; Wang, H.-L.; Zhou, L.; Zou, P.-P.; Wang, C. The Estimation and Experiment of Axial Force in Deep Well Pump Basing on Numerical Simulation. Int. J. Mod. Educ. Comput. Sci. 2010, 2, 53-61. [CrossRef]

(C) 2017 by the authors. Licensee MDPI, Basel, Switzerland. This article is an open access article distributed under the terms and conditions of the Creative Commons Attribution NonCommercial NoDerivatives (CC BY-NC-ND) license (https://creativecommons.org/licenses/by-nc-nd/4.0/). 